
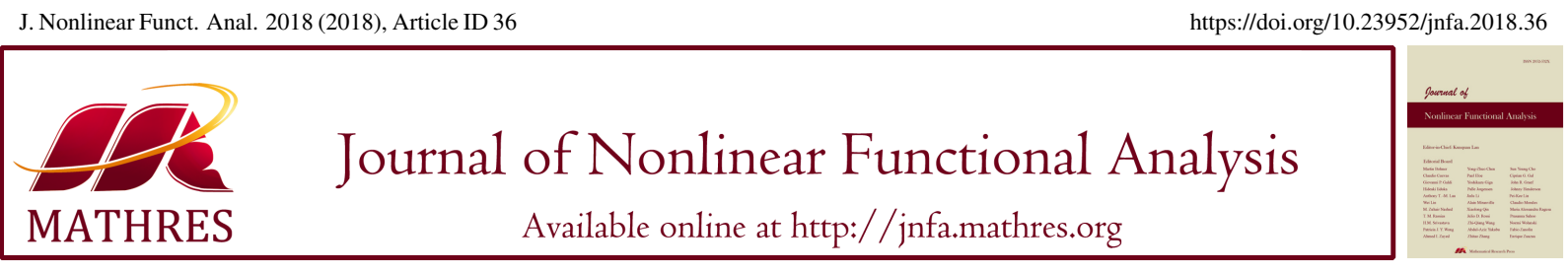

\title{
MULTI-TERM FRACTIONAL DIFFERENTIAL EQUATIONS AND INCLUSIONS WITH GENERALIZED NONLOCAL FRACTIONAL INTEGRO-DIFFERENTIAL BOUNDARY CONDITIONS
}

\author{
BASHIR AHMAD ${ }^{1, *}$, SOTIRIS K. NTOUYAS ${ }^{2,1}$, AHMED ALSAEDI $^{1}$, MADEAHA ALGHANMI ${ }^{1}$ \\ ${ }^{1}$ Nonlinear Analysis and Applied Mathematics (NAAM)-Research Group, Department of Mathematics, \\ Faculty of Science, King Abdulaziz University, P.O. Box 80203, Jeddah 21589, Saudi Arabia \\ ${ }^{2}$ Department of Mathematics, University of Ioannina, 45110 Ioannina, Greece
}

\begin{abstract}
In this paper, we study a new class of boundary value problems involving multiple fractional derivatives of Caputo type and generalized nonlocal fractional integro-differential boundary conditions. For the single-valued case, two existence results are obtained by means of nonlinear alternative of the Leray-Schauder type and the Krasnoselski's fixed point theorem, while the uniqueness of solutions is established by applying the contraction mapping principle. For the multi-valued case, two existence results are obtained by means of the Krasnoselski's multi-valued fixed point theorem and nonlinear alternative for contractive mappings. Examples illustrating the main results are also presented.

Keywords. Fractional differential equations; Caputo fractional derivative; Generalized fractional integral; Existence; Fixed point.
\end{abstract}

2010 Mathematics Subject Classification. 34A08, 34A12, 34A60.

\section{INTRODUCTION}

Boundary value problems of fractional differential equations involving different kinds of boundary conditions have been extensively investigated by many authors during the last few decades. The area of investigation for these problems includes the existence and the uniqueness of solutions, stability and oscillatory properties, analytic and numerical methods. The study of nonlinear boundary value problems is important as it helps to determine the effect of nonlinearity on the solutions of these problems. The interest in the subject of fractional calculus owes to the effective application of fractional-order differential and integral operators in the mathematical modeling of real world phenomena occurring in scientific and engineering disciplines. The mathematical models based on the tools of fractional calculus

\footnotetext{
${ }^{*}$ Corresponding author.

E-mail addresses: bashirahmad_qau@yahoo.com (B. Ahmad), sntouyas@uoi.gr (S.K. Ntouyas), aalsaedi@hotmail.com (A. Alsaedi), madeaha@hotmail.com (M. Alghanmi).

Received July 4, 2018; Accepted October 9, 2018.
}

(C)2018 Journal of Nonlinear Functional Analysis 
are found to be more realistic than the ones relying on classical calculus. One can also find applications of the subject in financial and economic issues, and social sciences. For a detailed description of the subject, we refer the reader to, for instance, the monographs by Kilbas, Srivastava and Trujillo [1], Lakshmikantham, Leela and Devi [2], Podlubny [3], Diethelm [4], Ahmad et al. [5] and the papers $[6,7,8,9,10,11,12,13,14,15,16,17,18,19]$.

In this paper, we introduce a new boundary value problem of fractional differential equations involving multiple Caputo type fractional derivatives and generalize nonlocal fractional integro-differential boundary conditions. To be more precise, we consider the following problem:

$$
\left\{\begin{array}{l}
D^{\alpha}\left[D^{\beta} y(t)-g(t, y(t))\right]=f(t, y(t)), t \in[0, T], \\
y(0)=0, D^{p} y(\eta)=\sum_{i=1}^{n} \lambda_{i}{ }^{\rho_{i}} I_{\theta_{i}, \kappa_{i}}^{\gamma_{i}, \delta_{i}} y\left(\xi_{i}\right), \eta, \xi_{i} \in(0, T),
\end{array}\right.
$$

where $D^{\chi}$ is the Caputo fractional derivatives of order $\chi \in(0,1), \chi=\alpha, \beta, p,{ }^{\rho_{i}} I_{\theta_{i}, \kappa_{i}}^{\gamma_{i}, \delta_{i}}$ is the generalized (Katugampola) fractional integral with $\gamma_{i}>0, \rho_{i}, \theta_{i}, \delta_{i}, \kappa_{i}, \lambda_{i} \in \mathbb{R}, i=1,2, \ldots, n$ [20], and $f, g$ : $[0, T] \times \mathbb{R} \rightarrow \mathbb{R}$ are given continuous functions. Here it is imperative to note that the integral boundary conditions play a crucial role in studying blood flow problems [21] and bacterial self-regularization [22]. The concept of nonlocal boundary conditions occupies a central role when physical, chemical or other processes depend on the interior positions (non-fixed points or segments) of the domain, for instance, see [23] and the references therein. Existence and uniqueness results for the problem (1.1) are established by applying the standard fixed point theorems.

We also investigate the multi-valued analogue of problem (1.1) given by

$$
\left\{\begin{array}{l}
D^{\alpha}\left[D^{\beta} y(t)-g(t, y(t))\right] \in F(t, y(t)), t \in[0, T], \\
y(0)=0, D^{p} y(\eta)=\sum_{i=1}^{n} \lambda_{i} \rho_{i} I_{\theta_{i}, \kappa_{i}}^{\gamma_{i}, \delta_{i}} y\left(\xi_{i}\right), \eta, \xi_{i} \in(0, T),
\end{array}\right.
$$

where $F:[0, T] \times \mathbb{R} \rightarrow \mathscr{P}(\mathbb{R})$ is a multivalued function $(\mathscr{P}(\mathbb{R})$ is the family of all nonempty subjects of $\mathbb{R})$.

The rest of the paper is organized as follows. In Section 2, we recall some basic concepts of fractional calculus and auxiliary results. The existence and uniqueness results for problem (1.1) are discussed in Section 3, while examples illustrating these results are presented in Section 4. The existence results for problem (1.2) are presented in Section 5. The paper concludes with an interesting "Discussion" Section.

\section{PRELIMINARIES}

Let us recall some preliminaries ideas of fractional calculus related to our proposed study. For general references, see the monographs [1,3]. For $c \in \mathbb{R}, 1 \leq p \leq \infty$, let $X_{c}^{p}(a, b)$ denote the space of all complexvalued Lebesgue measurable functions $\phi$ on $(a, b)$ with $\|\phi\|_{X_{c}^{p}}<\infty$, and

$$
\|\phi\|_{X_{c}^{p}}=\left(\int_{a}^{b}\left|x^{c} \phi(x)\right|^{p} \frac{d x}{x}\right)^{1 / p} \quad 1 \leq p<\infty .
$$

Definition 2.1. [20] Let $f \in X_{c}^{p}(a, b), \gamma, \delta>0$ and $\rho, \theta, \kappa \in \mathbb{R}$. Then the generalized fractional integral is defined by

$$
\left({ }^{\rho} I_{\theta, \kappa}^{\gamma, \delta} f\right)(t)=\frac{\rho^{1-\delta} t^{\kappa}}{\Gamma(\gamma)} \int_{0}^{t} \frac{s^{\rho(\theta+1)-1}}{\left(t^{\rho}-s^{\rho}\right)^{1-\gamma}} f(s) d s, 0<t<b
$$


Lemma 2.2. Let $\gamma, \delta>0$ and $\theta, \kappa, \rho \in \mathbb{R}$. Then

$$
\rho I_{\theta, \kappa}^{\gamma, \delta} t^{q}=\frac{t^{\kappa+\rho(\theta+\gamma)+q}}{\rho^{\delta}} \frac{\Gamma\left(\theta+\frac{q}{\rho}+1\right)}{\Gamma\left(\theta+\frac{q}{\rho}+\gamma+1\right)} .
$$

Definition 2.3. If $f \in C^{m}[a, b]$, then the Caputo fractional derivative $D_{a}^{\alpha}$ of order $\alpha \in \mathbb{R}(m-1<\alpha<$ $m, m \in \mathbb{N}$ ) is defined as

$$
D_{a}^{\alpha} f(t)=I_{a}^{1-\alpha} f^{(m)}(t)=\frac{1}{\Gamma(m-\alpha)} \int_{a}^{t}(t-s)^{m-1-\alpha} f^{(m)}(s) d s .
$$

Lemma 2.4. Let

$$
\Omega:=\frac{\eta^{\beta-p}}{\Gamma(1+\beta-p)}-\sum_{i=1}^{n} \lambda_{i} \frac{1}{\Gamma(\beta+1)} \frac{\xi_{i}^{\kappa_{i}+\rho_{i}\left(\theta_{i}+\gamma_{i}\right)+\beta}}{\rho_{i}^{\delta_{i}}} \frac{\Gamma\left(\theta_{i}+\frac{\beta}{\rho_{i}}+1\right)}{\Gamma\left(\theta_{i}+\frac{\beta}{\rho_{i}}+\gamma_{i}+1\right)} \neq 0
$$

The function y is a solution of problem (1.1) if and only if

$$
\begin{aligned}
y(t) & =J^{\beta} g(s, y(s))(t)+J^{\alpha+\beta} f(s, y(s))(t) \\
& +\frac{t^{\beta}}{\Gamma(\beta+1)} \frac{1}{\Omega}\left\{\sum_{i=1}^{n} \lambda_{i}{ }^{\rho_{i}} I_{\theta_{i}, \kappa_{i}}^{\gamma_{i}, \delta_{i}}\left(J^{\beta} g(s, y(s))+J^{\alpha+\beta} f(s, y(s))\right)\left(\xi_{i}\right)\right. \\
& \left.-J^{\beta-p} g(s, y(s))(\eta)-J^{\alpha+\beta-p} f(s, y(s))(\eta)\right\} .
\end{aligned}
$$

Proof. The solution of the differential equation in (1.1) can be written as

$$
y(t)=J^{\beta} g(s, y(s))(t)+J^{\alpha+\beta} f(s, y(s))(t)+\frac{t^{\beta}}{\Gamma(\beta+1)} c_{1}+c_{2},
$$

where $c_{1}$ and $c_{2} \in \mathbb{R}$ are arbitrary constants. From the first boundary condition in (1.1), we find that $c_{2}=0$. Taking Caputo fractional derivative of both sides of (2.4), we get

$$
D^{p} y(t)=J^{\beta-p} g(s, y(s))(t)+J^{\alpha+\beta-p} f(s, y(s))(t)+\frac{t^{\beta-p}}{\Gamma(\beta-p+1)} c_{1} .
$$

Applying the generalized fractional integral of order $\delta_{i}, \gamma_{i}>0$ to (2.4) yields

$$
\begin{aligned}
{ }^{\rho_{i}} I_{\theta_{i}, \kappa_{i}}^{\gamma_{i}, \delta_{i}} y(t)= & { }_{i} I_{\theta_{i}, \kappa_{i}}^{\gamma_{i}, \delta_{i}}\left(I^{\beta} g(s, y(s))+J^{\alpha+\beta} f(s, y(s))\right)(t) \\
& +c_{1} \frac{1}{\Gamma(1+\beta)} \frac{t^{\kappa_{i}+\rho_{i}\left(\theta_{i}+\gamma_{i}\right)+\beta}}{\rho_{i}^{\delta_{i}}} \frac{\Gamma\left(\theta_{i}+\frac{\beta}{\rho_{i}}+1\right)}{\Gamma\left(\theta_{i}+\frac{\beta}{\rho_{i}}+\gamma_{i}+1\right)} .
\end{aligned}
$$

Using (2.5) and (2.6) in the second boundary condition of (1.1), we obtain

$$
\begin{aligned}
c_{1}= & \frac{1}{\Omega}\left\{\sum_{i=1}^{n} \lambda_{i} \rho_{i} I_{\theta_{i}, \kappa_{i}}^{\gamma_{i}, \delta_{i}}\left(I^{\beta} g(s, y(s))+J^{\alpha+\beta} f(s, y(s))\right)\left(\xi_{i}\right)\right. \\
& \left.-J^{\beta-p} g(s, y(s))(\eta)-J^{\alpha+\beta-p} f(s, y(s))(\eta)\right\} .
\end{aligned}
$$

Inserting the values of $c_{1}$ and $c_{2}$ in (2.4), we get the solution (1.1). The converse follows by direct computation. This completes the proof. 


\section{EXISTENCE AND UNIQUENESS RESULTS FOR PROBLEM (1.1)}

Let $\mathscr{C}=C([0, T], \mathbb{R})$ denote the Banach space of all continuous functions from $[0, T]$ to $\mathbb{R}$ endowed with the norm defined by $\|y\|=\sup _{t \in[0, T]}|x(t)|$. Using Lemma 2.4, we define an operator $\mathscr{N}: \mathscr{C} \rightarrow \mathscr{C}$ as

$$
\begin{aligned}
\mathscr{N} y(t) & =J^{\beta} g(s, y(s))(t)+J^{\alpha+\beta} f(s, y(s))(t) \\
& +\frac{t^{\beta}}{\Gamma(\beta+1)} \frac{1}{\Omega}\left\{\sum_{i=1}^{n} \lambda_{i}{ }^{\rho_{i}} I_{\theta_{i}, \kappa_{i}}^{\gamma_{i}, \delta_{i}}\left(J^{\beta} g(s, y(s))+J^{\alpha+\beta} f(s, y(s))\right)\left(\xi_{i}\right)\right. \\
& \left.-J^{\beta-p} g(s, y(s))(\eta)-J^{\alpha+\beta-p} f(s, y(s))(\eta)\right\} .
\end{aligned}
$$

It should be noticed that problem (1.1) has solutions if and only if the operator $\mathscr{N}$ has fixed points.

In the following, for brevity, we use the notations:

$$
\Lambda_{1}=\frac{T^{\beta}}{\Gamma(\beta+1)}\left(1+\frac{1}{|\Omega|}\left\{\sum_{i=1}^{n} \lambda_{i} \frac{\xi_{i}^{\kappa_{i}+\rho_{i}\left(\theta_{i}+\gamma_{i}\right)+\beta}}{\rho_{i}^{\delta_{i}}} \frac{\Gamma\left(\theta_{i}+\frac{\beta}{\rho_{i}}+1\right)}{\Gamma\left(\gamma_{i}+\theta_{i}+\frac{\beta}{\rho_{i}}+1\right)}+\frac{\eta^{\beta-p}}{\Gamma(\beta-p+1)}\right\}\right)
$$

and

$$
\begin{aligned}
\Lambda_{2} & =\frac{T^{\alpha+\beta}}{\Gamma(\alpha+\beta+1)}+\frac{T^{\beta}}{|\Omega| \Gamma(\beta+1)}\left(\sum_{i=1}^{n} \lambda_{i} \frac{\xi_{i}^{\kappa_{i}+\rho_{i}\left(\theta_{i}+\gamma_{i}\right)+\alpha+\beta}}{\rho_{i}^{\delta_{i}}} \frac{\Gamma\left(\theta_{i}+\frac{\alpha+\beta}{\rho_{i}}+1\right)}{\Gamma\left(\gamma_{i}+\theta_{i}+\frac{\alpha+\beta}{\rho_{i}}+1\right)}\right. \\
& \left.+\frac{\eta^{\alpha+\beta-p}}{\Gamma(\alpha+\beta-p+1)}\right) .
\end{aligned}
$$

Now we are in a position to present our main results. Our first existence result is based on the LeraySchauder nonlinear alternative.

Lemma 3.1. (Nonlinear alternative for single valued maps) [24]. Let $E$ be a Banach space and let $C$ be a closed, convex subset of $E$. Let $U$ be an open subset of $C$ and $0 \in U$. Suppose that $F: \bar{U} \rightarrow C$ is a continuous, compact (that is, $F(\bar{U})$ is a relatively compact subset of $C$ ) map. Then either

(i) F has a fixed point in $\bar{U}$, or

(ii) there is a $u \in \partial U$ (the boundary of $U$ in $C$ ) and $\lambda \in(0,1)$ with $u=\lambda F(u)$.

Theorem 3.2. Assume that the following hypotheses hold:

$\left(H_{1}\right) f, g:[0, T] \times \mathbb{R} \rightarrow \mathbb{R}$ are continuous functions;

$\left(H_{2}\right)$ there exist constants $d_{1}<1 / \Lambda_{1}$ and $d_{2} \geq 0$ such that

$$
|g(t, u)| \leq d_{1}\|u\|+d_{2}, \quad t \in[0, T], u \in \mathbb{R} ;
$$

$\left(H_{3}\right)$ there exist a continuous nondecreasing function $\psi:[0, \infty) \rightarrow(0, \infty)$ and a function $p \in C\left([0, T], \mathbb{R}^{+}\right)$ such that

$$
|f(t, u)| \leq p(t) \psi(\|u\|) \text { for each }(t, u) \in[0, T] \times \mathbb{R}
$$

$\left(H_{4}\right)$ there exists a constant $M>0$ such that

$$
\frac{\left(1-d_{1} \Lambda_{1}\right) M}{d_{2} \Lambda_{1}+\Lambda_{2}\|p\| \psi(M)}>1
$$

where $\Lambda_{1}$ and $\Lambda_{2}$ are respectively given by (3.2) and (3.3).

Then problem (1.1) has at least one solution on $[0, T]$. 
Proof. We complete the proof in several steps. First of all, it will be shown that the operator $\mathscr{N}$ : $C([0, T], \mathbb{R}) \rightarrow C([0, T], \mathbb{R})$ defined by (3.1) is continuous and completely continuous.

Step 1: $\mathscr{N}$ is continuous.

Let $\left\{y_{n}\right\}$ be a sequence such that $y_{n} \rightarrow y$ in $C([0, T], \mathbb{R})$. Then

$$
\begin{aligned}
& \left|\mathscr{N}\left(y_{n}\right)(t)-\mathscr{N}(y)(t)\right| \\
\leq \quad & J^{\beta}\left|g\left(s, y_{n}(s)\right)-g(s, y(s))\right|(t)+J^{\alpha+\beta}\left|f\left(s, y_{n}(s)\right)-f(s, y(s))\right|(t) \\
& +\frac{T^{\beta}}{|\Omega| \Gamma(\beta+1)}\left\{\sum_{i=1}^{n} \lambda_{i}{ }^{\rho_{i}} I_{\theta_{i}, \kappa_{i}}^{\gamma_{i}, \delta_{i}}\left(J^{\beta}\left|g\left(s, y_{n}(s)\right)-g(s, y(s))\right|\right)\left(\xi_{i}\right)\right. \\
& +\sum_{i=1}^{n} \lambda_{i}{ }^{\rho_{i}} I_{\theta_{i}, \kappa_{i}}^{\gamma_{i}, \delta_{i}}\left(J^{\alpha+\beta}\left|f\left(s, y_{n}(s)\right)-f(s, y(s))\right|\right)\left(\xi_{i}\right) \\
& \left.+J^{\beta-p}\left|g\left(s, y_{n}(s)\right)-g(s, y(s))\right|(\eta)+J^{\alpha+\beta-p}\left|f\left(s, y_{n}(s)\right)-f(s, y(s))\right|(\eta)\right\} \\
\leq & \Lambda_{1}\left\|g\left(\cdot, y_{n}\right)-g(\cdot, y)\right\|+\Lambda_{2}\left\|f\left(\cdot, y_{n}\right)-f(\cdot, y)\right\| .
\end{aligned}
$$

Since $f, g$ are continuous functions, therefore, we have

$$
\left\|\mathscr{N}\left(y_{n}\right)-\mathscr{N}(y)\right\| \leq \Lambda_{1}\left\|g\left(\cdot, y_{n}\right)-g(\cdot, y)\right\|+\Lambda_{2}\left\|f\left(\cdot, y_{n}\right)-f(\cdot, y)\right\| \rightarrow 0,
$$

as $n \rightarrow \infty$.

Step 2: $\mathscr{N}$ maps bounded sets into bounded sets in $C([0, T], \mathbb{R})$.

Indeed, it is enough to show that for any $\theta>0$ there exists a positive constant $\tilde{\ell}$ such that, for each $y \in B_{\theta}=\{y \in C([0, T], \mathbb{R}):\|y\| \leq \theta\},\|N(y)\| \leq \tilde{\ell}$. By $\left(H_{2}\right)$ and $\left(H_{3}\right)$, for each $t \in[0, T]$, we have

$$
\begin{aligned}
|\mathscr{N}(y)(t)| \leq & J^{\beta}|g(s, y(s))|(t)+J^{\alpha+\beta}|f(s, y(s))|(t) \\
& +\frac{t^{\beta}}{|\Omega| \Gamma(\beta+1)}\left\{\sum_{i=1}^{n} \lambda_{i}{ }^{\rho_{i}} I_{\theta_{i}, K_{i}}^{\gamma_{i}, \delta_{i}}\left(J^{\beta}|g(s, y(s))|\right)\left(\xi_{i}\right)\right. \\
& +\sum_{i=1}^{n} \lambda_{i}{ }^{\rho_{i}} I_{\theta_{i}, \kappa_{i}}^{\gamma_{i}, \delta_{i}}\left(J^{\alpha+\beta}|f(s, y(s))|\right)\left(\xi_{i}\right) \\
& \left.+J^{\beta-p}|g(s, y(s))|(\eta)+J^{\alpha+\beta-p}|f(s, y(s))|(\eta)\right\} \\
\leq & \left(d_{1}\|y\|+d_{2}\right) \frac{T^{\beta}}{\Gamma(\beta+1)}+\|p\| \psi(\|y\|) \frac{T^{\alpha+\beta}}{\Gamma(\alpha+\beta+1)} \\
& +\frac{T^{\beta}}{|\Omega| \Gamma(\beta+1)}\left\{\sum_{i=1}^{n} \lambda_{i}\left(d_{1}\|y\|+d_{2}\right) \frac{\xi_{i}^{\kappa_{i}+\rho_{i}\left(\theta_{i}+\gamma_{i}\right)+\beta}}{\rho_{i}^{\delta_{i}}} \frac{\Gamma\left(\theta_{i}+\frac{\beta}{\rho_{i}}+1\right)}{\Gamma\left(\gamma_{i}+\theta_{i}+\frac{\beta}{\rho_{i}}+1\right)}\right. \\
& +\sum_{i=1}^{n} \lambda_{i}\|p\| \psi(\|y\|) \frac{\xi_{i}^{\kappa_{i}+\rho_{i}\left(\theta_{i}+\gamma_{i}\right)+\alpha+\beta}}{\rho_{i}^{\delta_{i}}} \frac{\Gamma\left(\theta_{i}+\frac{\alpha+\beta}{\rho_{i}}+1\right)}{\Gamma\left(\gamma_{i}+\theta_{i}+\frac{\alpha+\beta}{\rho_{i}}+1\right)} \\
& \left.+\left(d_{1}\|y\|+d_{2}\right) \frac{\eta^{\beta-p}}{\Gamma(\beta-p+1)}+\|p\| \psi(\|y\|) \frac{\eta^{\alpha+\beta-p}}{\Gamma(\alpha+\beta-p+1)}\right\}
\end{aligned}
$$


which, on taking the norm for $t \in[0, T]$, and using the notations (3.2) and (3.3), yields

$$
\|\mathscr{N}(y)\| \leq \Lambda_{1}\left(d_{1} \theta+d_{2}\right)+\Lambda_{2}\|p\| \psi(\theta):=\tilde{\ell} .
$$

Step 3: $\mathscr{N}$ maps bounded sets into equicontinuous sets of $C([0, T], \mathbb{R})$.

Let $t_{1}, t_{2} \in[0, T], t_{1}<t_{2}, B_{\theta}$ be a bounded set of $C([0, T], \mathbb{R})$ as in Step 2 , and let $y \in B_{\theta}$. Then

$$
\begin{aligned}
& \left|\mathscr{N}(y)\left(t_{2}\right)-\mathscr{N}(y)\left(t_{1}\right)\right| \\
& \leq\left|\frac{1}{\Gamma(\beta)} \int_{0}^{t_{1}}\left[\left(t_{2}-s\right)^{\beta-1}-\left(t_{1}-s\right)^{\beta-1}\right] g(s, y(s)) d s+\frac{1}{\Gamma(\beta)} \int_{t_{1}}^{t_{2}}\left(t_{2}-s\right)^{\beta-1} g(s, y(s)) d s\right| \\
& +\mid \frac{1}{\Gamma(\alpha+\beta)} \int_{0}^{t_{1}}\left[\left(t_{2}-s\right)^{\alpha+\beta-1}-\left(t_{1}-s\right)^{\alpha+\beta-1}\right] f(s, y(s)) d s \\
& +\frac{1}{\Gamma(\alpha+\beta)} \int_{t_{1}}^{t_{2}}\left(t_{2}-s\right)^{\alpha+\beta-1} f(s, y(s)) d s \\
& +\frac{\left|t_{2}^{\beta}-t_{1}^{\beta}\right|}{|\Omega| \Gamma(\beta+1)}\left\{\sum_{i=1}^{n} \lambda_{i} \rho_{i} I_{\theta_{i}, \kappa_{i}, \delta_{i}}\left(J^{\beta}|g(s, y(s))|\right)\left(\xi_{i}\right)+\sum_{i=1}^{n} \lambda_{i} \rho_{i} I_{\theta_{i}, K_{i}}^{\gamma_{i}, \delta_{i}}\left(J^{\alpha+\beta}|f(s, y(s))|\right)\left(\xi_{i}\right)\right. \\
& \left.+J^{\beta-p}|g(s, y(s))|(\eta)+J^{\alpha+\beta-p}|f(s, y(s))|(\eta)\right\} \\
& \leq \frac{d_{1} \theta+d_{2}}{\Gamma(\beta+1)}\left[t_{2}^{\beta}-t_{1}^{\beta}+2\left(t_{2}-t_{1}\right)^{\beta}\right]+\frac{\|p\| \psi(\theta)}{\Gamma(\alpha+\beta+1)}\left[t_{2}^{\alpha+\beta}-t_{1}^{\alpha+\beta}+2\left(t_{2}-t_{1}\right)^{\alpha+\beta}\right] \\
& +\frac{\left|t_{2}^{\beta}-t_{1}^{\beta}\right|}{|\Omega| \Gamma(\beta+1)}\left\{\left(\sum_{i=1}^{n} \lambda_{i} \frac{\xi_{i}^{\kappa_{i}+\rho_{i}\left(\theta_{i}+\gamma_{i}\right)+\beta}}{\rho_{i}^{\delta_{i}}} \frac{\Gamma\left(\theta_{i}+\frac{\beta}{\rho_{i}}+1\right)}{\Gamma\left(\gamma_{i}+\theta_{i}+\frac{\beta}{\rho_{i}}+1\right)}+\frac{\eta^{\beta-p}}{\Gamma(\beta-p+1)}\right)\left(d_{1} \theta+d_{2}\right)\right. \\
& \left.+\left(\sum_{i=1}^{n} \lambda_{i} \frac{\xi_{i}^{\kappa_{i}+\rho_{i}\left(\theta_{i}+\gamma_{i}\right)+\alpha+\beta}}{\rho_{i}^{\delta_{i}}} \frac{\Gamma\left(\theta_{i}+\frac{\alpha+\beta}{\rho_{i}}+1\right)}{\Gamma\left(\gamma_{i}+\theta_{i}+\frac{\alpha+\beta}{\rho_{i}}+1\right)}+\frac{\eta^{\alpha+\beta-p}}{\Gamma(\alpha+\beta-p+1)}\right)\|p\| \psi(\theta)\right\} .
\end{aligned}
$$

Clearly the right-hand side of the above inequality tends to zero independent of $y$ as $t_{1} \longrightarrow t_{2}$. In consequence of Steps 1 to 3, it follows by the Arzelá-Ascoli theorem that operator $\mathscr{N}: C([0, T], \mathbb{R}) \longrightarrow$ $C([0, T], \mathbb{R})$ is continuous and completely continuous.

Step 4: We show that there exists an open set $U \subseteq C([0, T], \mathbb{R})$ with $y \neq \mu \mathscr{N}(y)$ for $\mu \in(0,1)$ and $y \in \partial U$.

Let $y \in C([0, T], \mathbb{R})$ and $y=\mu N(y)$ for some $0<\mu<1$. Then, for each $t \in[0, T]$, we have

$$
\begin{aligned}
y(t)= & \mu J^{\beta} g(s, y(s))(t)+\mu J^{\alpha+\beta} f(s, y(s))(t) \\
& +\mu \frac{t^{\beta}}{\Gamma(\beta+1)} \frac{1}{\Omega}\left\{\sum_{i=1}^{n} \lambda_{i}{ }^{\rho_{i}} I_{\theta_{i}, \kappa_{i}}^{\gamma_{i}, \delta_{i}}\left(J^{\beta} g(s, y(s))+J^{\alpha+\beta} f(s, y(s))\right)\left(\xi_{i}\right)\right. \\
& \left.-J^{\beta-p} g(s, y(s))(\eta)-J^{\alpha+\beta-p} f(s, y(s))(\eta)\right\} .
\end{aligned}
$$

As in Step 2, for each $t \in[0, T]$, it can be established that

$$
|y(t)| \leq \Lambda_{1}\left(d_{1}\|y\|+d_{2}\right)+\Lambda_{2}\|p\| \psi(\|y\|),
$$

which can be expressed as

$$
\frac{\left(1-d_{1} \Lambda_{1}\right)\|y\|}{d_{2} \Lambda_{1}+\Lambda_{2}\|p\| \psi(\|y\|)} \leq 1 .
$$

In view of $\left(H_{4}\right)$, there exists $\mathrm{M}$ such that $\|y\| \neq \mathrm{M}$. Let us set

$$
U=\{y \in C([0, T], \mathbb{R}):\|y\|<\mathrm{M}\} .
$$


Note that the operator $\mathscr{N}: \bar{U} \rightarrow C([0, T], \mathbb{R})$ is continuous and completely continuous. From the choice of $U$, there is no $y \in \partial U$ such that $y=\mu \mathscr{N} y$ for some $\mu \in(0,1)$. Consequently, by the nonlinear alternative of Leray-Schauder type (Lemma 3.1), we deduce that $\mathscr{N}$ has a fixed point $y \in \bar{U}$ which is a solution of the problem (1.1). This completes the proof.

In the following result, we prove the uniqueness of solutions for the problem (1.1) by using Banach's contraction mapping principle.

Theorem 3.3. Assume that the following conditions hold:

$\left(A_{1}\right)$ there exists a nonnegative constant $k$ such that $|g(t, u)-g(t, v)| \leq k\|u-v\|$ for $t \in[0, T]$ and every $u, v \in \mathbb{R}$

$\left(A_{2}\right)$ there exists $\ell>0$ such that $|f(t, u)-f(t, v)| \leq \ell\|u-v\|$, for $t \in[0, T]$ and every $u, v \in \mathbb{R}$.

If

$$
k \Lambda_{1}+\ell \Lambda_{2}<1,
$$

where $\Lambda_{1}, \Lambda_{2}$ are defined by (3.2) and (3.3) respectively, then there exists a unique solution for the problem (1.1) on $[0, T]$.

Proof. With $\Lambda_{1}$ and $\Lambda_{2}$ respectively given by (3.2) and (3.3), we fix

$$
r \geq \frac{\Lambda_{1} g_{0}+\Lambda_{2} f_{0}}{1-k \Lambda_{1}-\ell \Lambda_{2}}, g_{0}=\sup _{t \in[0, T]}|g(t, 0)|, f_{0}=\sup _{t \in[0, T]}|f(t, 0)|,
$$

and show that $\mathscr{N} B_{r} \subset B_{r}$, where operator $\mathscr{N}: C([0, T], \mathbb{R}) \rightarrow C([0, T], \mathbb{R})$ is given by (3.1) and $B_{r}=$ $\{y \in C([0, T], \mathbb{R}):\|y\| \leq r\}$. For $x \in B_{r}$, using $\left(A_{1}\right)$ and $\left(A_{2}\right)$, we have

$$
\begin{aligned}
|\mathscr{N}(y)(t)| \leq & J^{\beta}[|g(s, y(s))-g(s, 0)|+|g(s, 0)|](t) \\
& +J^{\alpha+\beta}[|f(s, y(s))-f(s, 0)|+|f(s, 0)|](t) \\
& +\frac{t^{\beta}}{|\Omega| \Gamma(\beta+1)}\left\{\sum_{i=1}^{n} \lambda_{i}{ }^{\rho_{i}} I_{\theta_{i}, \kappa_{i}}^{\gamma_{i}, \delta_{i}}\left(J^{\beta}[|g(s, y(s))-g(s, 0)|+|g(s, 0)|]\right)\left(\xi_{i}\right)\right. \\
& +\sum_{i=1}^{n} \lambda_{i}{ }^{\rho_{i}} I_{\theta_{i}, \kappa_{i}, \delta_{i}}\left(J^{\alpha+\beta}[|f(s, y(s))-f(s, 0)|+|f(s, 0)|]\right)\left(\xi_{i}\right) \\
& +J^{\beta-p}[|g(s, y(s))-g(s, 0)|+|g(s, 0)|](\eta) \\
& \left.+J^{\alpha+\beta-p}[|f(s, y(s))-f(s, 0)|+|f(s, 0)|](\eta)\right\} \\
\leq & \left(k\|y\|+g_{0}\right) \frac{T^{\beta}}{\Gamma(\beta+1)}+\left(\ell\|y\|+f_{0}\right) \frac{T^{\alpha+\beta}}{\Gamma(\alpha+\beta+1)} \\
& +\frac{T^{\beta}}{|\Omega| \Gamma(\beta+1)}\left\{\sum_{i=1}^{n} \lambda_{i}\left(k\|y\|+g_{0}\right) \frac{\xi_{i}^{\kappa_{i}+\rho_{i}\left(\theta_{i}+\gamma_{i}\right)+\beta}}{\rho_{i}^{\delta_{i}}} \frac{\Gamma\left(\theta_{i}+\frac{\beta}{\rho_{i}}+1\right)}{\Gamma\left(\gamma_{i}+\theta_{i}+\frac{\beta}{\rho_{i}}+1\right)}\right. \\
& +\sum_{i=1}^{n} \lambda_{i}\left(\ell\|y\|+f_{0}\right) \frac{\xi_{i}^{\kappa_{i}+\rho_{i}\left(\theta_{i}+\gamma_{i}\right)+\alpha+\beta}}{\rho_{i}^{\delta_{i}}} \frac{\Gamma\left(\theta_{i}+\frac{\alpha+\beta}{\rho_{i}}+1\right)}{\Gamma\left(\gamma_{i}+\theta_{i}+\frac{\alpha+\beta}{\rho_{i}}+1\right)} \\
& \left.+\left(k\|y\|+g_{0}\right) \frac{\eta^{\beta-p}}{\Gamma(\beta-p+1)}+\left(\ell\|y\|+f_{0}\right) \frac{\eta^{\alpha+\beta-p}}{\Gamma(\alpha+\beta-p+1)}\right\} \\
\leq \quad & \Lambda_{1}\left(k r+g_{0}\right)+\Lambda_{2}\left(\ell r+f_{0}\right) \leq r,
\end{aligned}
$$


which, on taking the norm for $t \in[0, T]$, implies that $\|\mathscr{N}(y)\| \leq r$. Thus $\mathscr{N}$ maps $B_{r}$ into itself.

In order to show that $\mathscr{N}$ is a contraction, let $y, z \in C([0, T], \mathbb{R})$. Using (3.2) and (3.3), we get

$$
\begin{aligned}
& |\mathscr{N}(y)(t)-\mathscr{N}(z)(t)| \\
& \leq J^{\beta}|g(s, y(s))-g(s, z(s))|(t)+J^{\alpha+\beta}|f(s, y(s))-f(s, z(s))|(t) \\
& +\frac{T^{\beta}}{|\Omega| \Gamma(\beta+1)}\left\{\sum_{i=1}^{n} \lambda_{i} \rho_{i} I_{\theta_{i}, \kappa_{i}}^{\gamma_{i}, \delta_{i}}\left(J^{\beta}|g(s, y(s))-g(s, z(s))|\right)\left(\xi_{i}\right)\right. \\
& +\sum_{i=1}^{n} \lambda_{i}{ }^{\rho_{i}} I_{\theta_{i}, \kappa_{i}}^{\gamma_{i}, \delta_{i}}\left(J^{\alpha+\beta}|f(s, y(s))-f(s, z(s))|\right)\left(\xi_{i}\right) \\
& \left.+J^{\beta-p}|g(s, y(s))-g(s, z(s))|(\eta)+J^{\alpha+\beta-p}|f(s, y(s))-f(s, z(s))|(\eta)\right\} \\
& \leq k\|y-z\| \frac{T^{\beta}}{\Gamma(\beta+1)}+\ell\|y-z\| \frac{T^{\alpha+\beta}}{\Gamma(\alpha+\beta+1)} \\
& +\frac{T^{\beta}}{|\Omega| \Gamma(\beta+1)}\left\{\sum_{i=1}^{n} \lambda_{i} \frac{\xi_{i}^{\kappa_{i}+\rho_{i}\left(\theta_{i}+\gamma_{i}\right)+\beta}}{\rho_{i}^{\delta_{i}}} \frac{\Gamma\left(\theta_{i}+\frac{\beta}{\rho_{i}}+1\right)}{\Gamma\left(\gamma_{i}+\theta_{i}+\frac{\beta}{\rho_{i}}+1\right)} k\|y-z\|\right. \\
& +\sum_{i=1}^{n} \lambda_{i} \frac{\xi_{i}^{\kappa_{i}+\rho_{i}\left(\theta_{i}+\gamma_{i}\right)+\alpha+\beta}}{\rho_{i}^{\delta_{i}}} \frac{\Gamma\left(\theta_{i}+\frac{\alpha+\beta}{\rho_{i}}+1\right)}{\Gamma\left(\gamma_{i}+\theta_{i}+\frac{\alpha+\beta}{\rho_{i}}+1\right)} \ell\|y-z\| \\
& \left.+k\|y-z\| \frac{\eta^{\beta-p}}{\Gamma(\beta-p+1)}+\ell\|y-z\| \frac{\eta^{\alpha+\beta-p}}{\Gamma(\alpha+\beta-p+1)}\right\} \\
& =\left(k \Lambda_{1}+\ell \Lambda_{2}\right)\|y-z\| \text {. }
\end{aligned}
$$

Consequently, we obtain

$$
\|\mathscr{N}(y)-\mathscr{N}(z)\| \leq\left(k \Lambda_{1}+\ell \Lambda_{2}\right)\|y-z\|
$$

which, in view of (3.4), implies that $\mathscr{N}$ is a contraction. Hence $\mathscr{N}$ has a unique fixed point by the Banach's contraction principle. This, in turn, shows that problem (1.1) has a unique solution on $[0, T]$. The proof is completed.

Our final existence result is based on the Krasnoselskii's fixed point theorem.

Lemma 3.4. (Krasnoselskii's fixed point theorem) [25]. Let $S$ be a closed, bounded, convex and nonempty subset of a Banach space X. Let $\mathscr{Y}_{1}, \mathscr{Y}_{2}$ be the operators such that $(a) \mathscr{Y}_{1} s_{1}+\mathscr{Y}_{2} s_{2} \in S$ whenever $s_{1}, s_{2} \in S ;(b) \mathscr{Y}_{1}$ is compact and continuous; (c) $\mathscr{Y}_{2}$ is a contraction mapping. Then there exists $s_{3} \in S$ such that $s_{3}=\mathscr{Y}_{1} s_{3}+\mathscr{Y}_{2} s_{3}$.

Theorem 3.5. Assume that $\left(A_{1}\right)$ and $\left(H_{1}\right)$ hold. In addition, we assume that

$$
\left(H_{5}\right) \quad|g(t, x)| \leq \phi(t),|f(t, x)| \leq q(t), \quad \forall(t, x) \in[0, T] \times \mathbb{R}, \text { and } q, \phi \in C\left([0, T], \mathbb{R}^{+}\right) .
$$

Then problem (1.1) has at least one solution on $[0, T]$, provided that

$$
k \Lambda_{1}<1
$$


Proof. Let us split $\mathscr{N}: C([0, T], \mathbb{R}) \rightarrow C([0, T], \mathbb{R})$ defined by (3.1) as $\mathscr{N}=\mathscr{A}_{1}+\mathscr{A}_{2}$, where $\mathscr{A}_{1}$ and $\mathscr{A}_{2}$ are given by

$$
\begin{aligned}
\mathscr{A}_{1}(x)(t)= & J^{\beta} g(s, y(s))(t)+\frac{t^{\beta}}{\Gamma(\beta+1)} \frac{1}{\Omega}\left\{\sum_{i=1}^{n} \lambda_{i}{ }^{\rho_{i}} I_{\theta_{i}, \kappa_{i}}^{\gamma_{i}, \delta_{i}}\left(J^{\beta} g(s, y(s))\right)\left(\xi_{i}\right)\right. \\
& \left.-J^{\beta-p} g(s, y(s))(\eta)\right\},
\end{aligned}
$$

and

$$
\begin{aligned}
\mathscr{A}_{2}(x)(t)= & J^{\alpha+\beta} f(s, y(s))(t)+\frac{t^{\beta}}{\Gamma(\beta+1)} \frac{1}{\Omega}\left\{\sum_{i=1}^{n} \lambda_{i}{ }^{\rho_{i}} I_{\theta_{i}, \kappa_{i}}^{\gamma_{i}, \delta_{i}}\left(J^{\alpha+\beta} f(s, y(s))\right)\left(\xi_{i}\right)\right. \\
& \left.-J^{\alpha+\beta-p} f(s, y(s))(\eta)\right\} .
\end{aligned}
$$

Setting $\sup _{t \in[0, T]}|\phi(t)|=\|\phi\|, \sup _{t \in[0, T]}|q(t)|=\|q\|$ and choosing $\rho \geq\|\phi\| \Lambda_{1}+\|q\| \Lambda_{2}$, we consider $B_{\rho}=\{y \in C([0, T], \mathbb{R}):\|y\| \leq \rho\}$. For any $y, z \in B_{\rho}$, we have

$$
\begin{aligned}
\left|\mathscr{A}_{1} y(t)+\mathscr{A}_{2} z(t)\right| \leq & J^{\beta}|g(s, y(s))|(t)+J^{\alpha+\beta}|f(s, z(s))|(t) \\
& +\frac{t^{\beta}}{|\Omega| \Gamma(\beta+1)}\left\{\sum_{i=1}^{n} \lambda_{i}{ }^{\rho_{i}} I_{\theta_{i}, \kappa_{i}}^{\gamma_{i}, \delta_{i}}\left(J^{\beta}|g(s, y(s))|\right)\left(\xi_{i}\right)\right. \\
& +\sum_{i=1}^{n} \lambda_{i}{ }^{\rho_{i}} I_{\theta_{i}, \kappa_{i}}^{\gamma_{i}, \delta_{i}}\left(J^{\alpha+\beta}|f(s, z(s))|\right)\left(\xi_{i}\right) \\
& \left.+J^{\beta-p}|g(s, y(s))|(\eta)+J^{\alpha+\beta-p}|f(s, z(s))|(\eta)\right\} \\
\leq & \|\phi\| \Lambda_{1}+\|q\| \Lambda_{2} \leq \rho .
\end{aligned}
$$

Hence $\left\|\mathscr{A}_{1} y+\mathscr{A}_{2} z\right\| \leq \rho$, which shows that $\mathscr{A}_{1} y+\mathscr{A}_{2} z \in B_{\rho}$. In view of (3.5), it is easy to show that $\mathscr{A}_{1}$ is a contraction. Continuity of $f$ implies that the operator $\mathscr{A}_{2}$ is continuous. Also, $\mathscr{A}_{2}$ is uniformly bounded on $B_{\rho}$ as $\left\|\mathscr{A}_{2} y\right\| \leq\|q\| \Lambda_{2}$. Finally we prove the compactness of the operator $\mathscr{A}_{2}$. For that we define $\sup _{(t, y) \in[0, T] \times B_{\rho}}|f(t, y)|=\bar{f}<\infty$. Then, for $t_{1}, t_{2} \in[0, T], t_{1}<t_{2}$, we have

$$
\begin{aligned}
& \left|\mathscr{A}_{2} y\left(t_{2}\right)-\mathscr{A}_{2} y\left(t_{1}\right)\right| \\
\leq & \mid \frac{1}{\Gamma(\alpha+\beta)} \int_{0}^{t_{1}}\left[\left(t_{2}-s\right)^{\alpha+\beta-1}-\left(t_{1}-s\right)^{\alpha+\beta-1}\right] f(s, y(s)) d s \\
& +\frac{1}{\Gamma(\alpha+\beta)} \int_{t_{1}}^{t_{2}}\left(t_{2}-s\right)^{\alpha+\beta-1} f(s, y(s)) d s \mid \\
& +\frac{\left|t_{2}^{\beta}-t_{1}^{\beta}\right|}{|\Omega| \Gamma(\beta+1)}\left\{\sum_{i=1}^{n} \lambda_{i}{ }^{\rho_{i}} I_{\theta_{i}, \kappa_{i}, \delta_{i}}\left(J^{\alpha+\beta}|f(s, y(s))|\right)\left(\xi_{i}\right)+J^{\alpha+\beta-p}|f(s, y(s))|(\eta)\right\} \\
\leq & \frac{\bar{f}}{\Gamma(\alpha+\beta+1)}\left[t_{2}^{\alpha+\beta}-t_{1}^{\alpha+\beta}+2\left(t_{2}-t_{1}\right)^{\alpha+\beta}\right] \\
& +\frac{\left|t_{2}^{\beta}-t_{1}^{\beta}\right| \bar{f}}{|\Omega| \Gamma(\beta+1)}\left\{\sum_{i=1}^{n} \lambda_{i} \frac{\xi_{i}^{\kappa_{i}+\rho_{i}\left(\theta_{i}+\gamma_{i}\right)+\alpha+\beta}}{\rho_{i}^{\delta_{i}}} \frac{\Gamma\left(\theta_{i}+\frac{\alpha+\beta}{\rho_{i}}+1\right)}{\Gamma\left(\gamma_{i}+\theta_{i}+\frac{\alpha+\beta}{\rho_{i}}+1\right)}+\frac{\eta^{\alpha+\beta-p}}{\Gamma(\alpha+\beta-p+1)}\right\},
\end{aligned}
$$

which is independent of $y$ and tends to zero as $t_{2}-t_{1} \rightarrow 0$. Thus, $\mathscr{A}_{2}$ is equicontinuous. So $\mathscr{A}_{2}$ is relatively compact on $B_{\rho}$. Hence, by the Arzelá-Ascoli theorem, $\mathscr{A}_{2}$ is compact on $B_{\rho}$. Thus all the assumptions of Lemma 3.4 are satisfied. So the conclusion of Lemma 3.4 implies that problem (1.1) has at least one solution on $[0, T]$. 


\section{EXAMPLES}

In this section, we give examples to illustrate the usefulness of our main results. To this end, let us consider the problem

$$
\left\{\begin{array}{l}
D^{1 / 2}\left[D^{3 / 4} y(t)-g(t, y(t))\right]=f(t, y(t)), t \in[0,2] \\
y(0)=0, D^{1 / 4} y(3 / 4)=I^{1 / 3,1} y(1)+1 / 2^{2 / 5} I^{1 / 2,1 / 2} y(3 / 2),
\end{array}\right.
$$

where $\alpha=1 / 2, \beta=3 / 4, p=1 / 4, \gamma_{1}=1 / 3, \delta_{1}=1, \rho_{1}=1, \kappa_{1}=\theta_{1}=0, \lambda_{1}=1, \xi_{1}=1, \gamma_{2}=\delta_{2}=$ $1 / 2, \rho_{2}=2 / 5, \kappa_{2}=\theta_{2}=0, \lambda_{2}=1 / 2, \xi_{2}=3 / 2$ and

$$
f(t, y)=\frac{1}{2 \sqrt{900+t^{2}}} e^{-t}\left(\tan ^{-1} y+y+\frac{1}{8}\right) \text { and } g(t, y)=\frac{1}{30\left(1+t^{2}\right)} \sin y+\frac{e^{-t}}{25} .
$$

Using the given data, it is found that $|\Omega| \approx 0.7647229355, \Lambda_{1} \approx 7.999127836, \Lambda_{2} \approx 7.542328910$, where $\Omega, \Lambda_{1}$ and $\Lambda_{2}$ are respectively given by (2.2), (3.2) and (3.3).

(i) In order to illustrate Theorem 3.2, note that $d_{1}=1 / 30, d_{2}=1 / 25, \psi(\|y\|)=\|y\|+\frac{4 \pi+1}{8}, p(t)=$ $\frac{1}{2 \sqrt{900+t^{2}}} e^{-t}$. By the condition:

$$
\frac{\left(1-d_{1} \Lambda_{1}\right) M}{d_{2} \Lambda_{1}+\Lambda_{2}\|p\| \psi(M)}>1
$$

it follows that $M=0.8773635038$. Clearly the hypothesis of Theorem 3.2 holds and consequently there exists at least one solution for the problem (4.1).

(ii) It is easy to check that $f(t, x)$ and $g(t, x)$ satisfy the conditions $\left(A_{1}\right)$ and $\left(A_{2}\right)$ respectively with $\ell=1 / 30$ and $k=1 / 30$. Also

$$
k \Lambda_{1}+\ell \Lambda_{2} \approx 0.5180485582<1 \text {. }
$$

Thus all the conditions of Theorem 3.3 are satisfied. So, by the conclusion of Theorem 3.3, problem (4.1) has a unique solution on [0,2].

(iii) To illustrate Theorem 3.5, we take

$$
f(t, y)=\frac{1}{2 \sqrt{900+t^{2}}}\left(\tan ^{-1} y+e^{-t}\right) \text { and } g(t, y)=\frac{1}{30\left(1+t^{2}\right)} \sin y+\frac{e^{-t}}{25} .
$$

With $\phi(t)=\frac{1}{30\left(1+t^{2}\right)}+\frac{e^{-t}}{25}, q(t)=\frac{\left(\pi+2 e^{-t}\right)}{4 \sqrt{900+t^{2}}}$ and $k \Lambda_{1} \approx 0.2666375945<1$, the hypothesis of Theorem 3.5 is satisfied, which implies that there exists at least one solution for the problem (4.1) with $f(t, x)$ and $g(t, x)$ given by (4.2).

\section{EXISTENCE RESUlTS FOR PROBLEM (1.2)}

We begin this section with some background material on multi-valued maps related to our work [26, $27,28]$.

For a normed space $(X,\|\cdot\|)$, let $\mathscr{P}_{c l}(X)=\{Y \in \mathscr{P}(X): Y$ is closed $\}, \mathscr{P}_{b}(X)=\{Y \in \mathscr{P}(X)$ : $Y$ is bounded $\}, \mathscr{P}_{c p}(X)=\{Y \in \mathscr{P}(X): Y$ is compact $\}$ and $\mathscr{P}_{c p, c}(X)=\{Y \in \mathscr{P}(X): Y$ is compact and convex $\}$.

A multi-valued map $G: X \rightarrow \mathscr{P}(X)$ :

(i) is convex (closed) valued if $G(x)$ is convex (closed) for all $x \in X$;

(ii) is bounded on bounded sets if $G(\mathbb{B})=\cup_{x \in \mathbb{B}} G(x)$ is bounded in $X$ for all $\mathbb{B} \in \mathscr{P}_{b}(X)$ (i.e. $\left.\sup _{x \in \mathbb{B}}\{\sup \{|y|: y \in G(x)\}\}<\infty\right)$; 
(iii) is said to be upper semi-continuous (u.s.c.) on $X$ if for each $x_{0} \in X$, the set $G\left(x_{0}\right)$ is a nonempty closed subset of $X$, and if for each open set $N$ of $X$ containing $G\left(x_{0}\right)$, there exists an open neighborhood $\mathscr{N}_{0}$ of $x_{0}$ such that $G\left(\mathscr{N}_{0}\right) \subseteq N$;

(iv) $G$ is lower semi-continuous (l.s.c.) if the set $\{y \in X: G(y) \cap B \neq \emptyset\}$ is open for any open set $B$ in $E$;

(v) is said to be completely continuous if $G(\mathbb{B})$ is relatively compact for every $\mathbb{B} \in \mathscr{P}_{b}(X)$;

(vi) is said to be measurable if for every $y \in \mathbb{R}$, the function

$$
t \longmapsto d(y, G(t))=\inf \{|y-z|: z \in G(t)\}
$$

is measurable;

(vii) has a fixed point if there is $x \in X$ such that $x \in G(x)$. The fixed point set of the multivalued operator $G$ will be denoted by Fix $G$.

Definition 5.1. A multivalued map $F:[0, T] \times \mathbb{R} \rightarrow \mathscr{P}(\mathbb{R})$ is said to be Carathéodory if

(i) $t \longmapsto F(t, y)$ is measurable for each $y \in \mathbb{R}$;

(ii) $y \longmapsto F(t, y)$ is upper semicontinuous for almost all $t \in[0, T]$;

Further a Carathéodory function $F$ is said to be $L^{1}$-Carathéodory if

(iii) for each $\alpha>0$, there exists $\varphi_{\alpha} \in L^{1}\left([0, T], \mathbb{R}^{+}\right)$such that

$$
\|F(t, y)\|=\sup \{|v|: v \in F(t, y)\} \leq \varphi_{\alpha}(t)
$$

for all $y \in \mathbb{R}$ with $\|y\| \leq \alpha$ and for a. e. $t \in[0, T]$.

For each $y \in C([0, T], \mathbb{R})$, define the set of selections of $F$ by

$$
S_{F, y}:=\left\{v \in L^{1}([0, T], \mathbb{R}): v(t) \in F(t, y(t)) \text { for a.e. } t \in[0, T]\right\} .
$$

We define the graph of $G$ to be the set $\operatorname{Gr}(G)=\{(x, y) \in X \times Y, y \in G(x)\}$ and recall two useful results on closed graphs and upper-semicontinuity.

Lemma 5.2. ([26, Proposition 1.2]) If $G: X \rightarrow \mathscr{P}_{c l}(Y)$ is u.s.c., then $G r(G)$ is a closed subset of $X \times Y$; i.e., for every sequence $\left\{x_{n}\right\}_{n \in \mathbb{N}} \subset X$ and $\left\{y_{n}\right\}_{n \in \mathbb{N}} \subset Y$, if when $n \rightarrow \infty, x_{n} \rightarrow x_{*}, y_{n} \rightarrow y_{*}$ and $y_{n} \in G\left(x_{n}\right)$, then $y_{*} \in G\left(x_{*}\right)$. Conversely, if $G$ is completely continuous and has a closed graph, then it is upper semi-continuous.

Lemma 5.3. ([29]) Let $X$ be a Banach space. Let $F:[0, T] \times \mathbb{R} \rightarrow \mathscr{P}_{c p, c}(X)$ be an $L^{1}-$ Carathéodory multivalued map and let $\Theta$ be a linear continuous mapping from $L^{1}([0, T], X)$ to $C([0, T], X)$. Then the operator

$$
\Theta \circ S_{F}: C([0, T], X) \rightarrow \mathscr{P}_{c p, c}(C([0, T], X)), x \mapsto\left(\Theta \circ S_{F}\right)(x)=\Theta\left(S_{F, x, y}\right)
$$

is a closed graph operator in $C([0, T], X) \times C([0, T], X)$.

Lemma 5.4. ([30], Krasnoselskii's fixed point theorem). Let $X$ be a Banach space, $Y \in \mathscr{P}_{b, c l, c}(X)$ and $A, B: Y \rightarrow \mathscr{P}_{c p, c}(X)$ two multivalued operators. If the following conditions are satisfied

(i) $A y+B y \subset Y$ for all $y \in Y$;

(ii) A is contraction;

(iii) $B$ is u.s.c. and compact,

then, there exists $y \in Y$ such that $y \in A y+B y$. 
Definition 5.5. A function $y \in C^{2}([0, T], \mathbb{R})$ is a solution of problem (1.2) if $y(0)=0, D^{p} y(\eta)=$ $\sum_{i=1}^{n} \lambda_{i}{ }^{\rho_{i}} I_{\theta_{i}, \kappa_{i}}^{\gamma_{i}, \delta_{i}} y\left(\xi_{i}\right)$, and there exists a function $v \in L^{1}([0, T], \mathbb{R})$ such that $v(t) \in F(t, y(t))$ a.e. on $[0, T]$ and

$$
\begin{aligned}
y(t) & =I^{\beta} g(s, y(s))(t)+I^{\alpha+\beta} v(s)(t) \\
& +\frac{t^{\beta}}{\Gamma(\beta+1)} \frac{1}{\Omega}\left\{\sum_{i=1}^{n} \lambda_{i} \rho_{i} I_{\theta_{i}, \kappa_{i}}^{\gamma_{i}, \delta_{i}}\left(I^{\beta} g(s, y(s))+I^{\alpha+\beta} v(s)\right)\left(\xi_{i}\right)\right. \\
& \left.-I^{\beta-p} g(s, y(s))(\eta)-I^{\alpha+\beta-p} v(s)(\eta)\right\} .
\end{aligned}
$$

Theorem 5.6. Assume that $\left(A_{1}\right)$ with $k<\Lambda_{1}^{-1}$ and the following conditions hold:

$\left(B_{1}\right) F:[0, T] \times \mathbb{R} \rightarrow \mathscr{P}_{c p, c}(\mathbb{R})$ is $L^{1}$-Carathéodory multivalued map;

$\left(B_{2}\right)$ there exists a function $p \in C\left([0, T], \mathbb{R}^{+}\right)$such that

$$
\|F(t, y)\|_{\mathscr{P}}:=\sup \{|x|: x \in F(t, y)\} \leq p(t), \text { for each }(t, y) \in[0, T] \times \mathbb{R} .
$$

Then boundary value problem (1.2) has at least one solution on $[0, T]$.

Proof. Let us transform problem (1.2) into a fixed point problem by defining an operator $\mathscr{N}_{m}: \mathscr{C} \longrightarrow$ $\mathscr{P}(\mathscr{C})$ as follows:

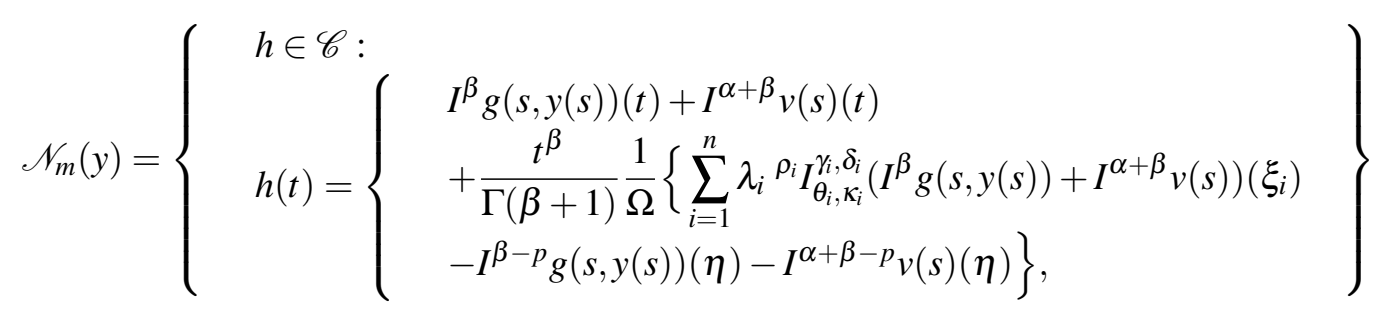

for $v \in S_{F, y}$.

Next we introduce operators $\mathscr{N}_{1}: \mathscr{C} \longrightarrow \mathscr{C}$ and $\mathscr{N}_{2}: \mathscr{C} \longrightarrow \mathscr{P}(\mathscr{C})$ by

$$
\begin{aligned}
& \mathscr{N}_{1} y(t)=I^{\beta} g(s, y(s))(t)+\frac{t^{\beta}}{\Gamma(\beta+1)} \frac{1}{\Omega}\left\{\sum_{i=1}^{n} \lambda_{i} \rho_{i} I_{\theta_{i}, \kappa_{i}}^{\gamma_{i}, \delta_{i}}\left(I^{\beta} g(s, y(s))\right)\left(\xi_{i}\right)\right. \\
& \left.-I^{\beta-p} g(s, y(s))(\eta)\right\}, \\
& \mathscr{N}_{2}(y)=\left\{\begin{array}{l}
h \in \mathscr{C}: \\
h(t)=I^{\alpha+\beta} v(s)(t)+\frac{t^{\beta}}{\Gamma(\beta+1)} \frac{1}{\Omega}\left\{\sum_{i=1}^{n} \lambda_{i} \rho_{i} I_{\theta_{i}, \kappa_{i}}^{\gamma_{i}, \delta_{i}}\left(I^{\alpha+\beta} v(s)\right)\left(\xi_{i}\right)\right\} \\
\left.-I^{\alpha+\beta-p} v(s)(\eta)\right\} .
\end{array}\right.
\end{aligned}
$$

Evidently $\mathscr{N}_{m}=\mathscr{N}_{1}+\mathscr{N}_{2}$. The proof will be complete once we establish that the operators $\mathscr{N}_{1}$ and $\mathscr{N}_{2}$ satisfy all the conditions of Lemma 5.4 on $[0, T]$. First, we show that the operators $\mathscr{N}_{1}$ and $\mathscr{N}_{2}$ define the multivalued operators $\mathscr{N}_{1}, \mathscr{N}_{2}: B_{r} \rightarrow \mathscr{P}_{c p, c}(\mathscr{C})$, where $B_{r}=\{y \in \mathscr{C}:\|y\| \leq r\}$ is a bounded set in $\mathscr{C}$. First we prove that $\mathscr{N}_{2}$ is compact-valued on $B_{r}$. Note that the operator $\mathscr{N}_{2}$ is equivalent to the composition $\mathscr{L} \circ S_{F}$, where $\mathscr{L}$ is the continuous linear operator on $L^{1}([0, T], \mathbb{R})$ into $\mathscr{C}$, defined by

$$
\mathscr{L}(v)(t)=I^{\alpha+\beta} v(s)(t)+\frac{t^{\beta}}{\Gamma(\beta+1)} \frac{1}{\Omega}\left\{\sum_{i=1}^{n} \lambda_{i}{ }^{\rho_{i}} I_{\theta_{i}, \kappa_{i}}^{\gamma_{i}, \delta_{i}}\left(I^{\alpha+\beta} v(s)\right)\left(\xi_{i}\right)-I^{\alpha+\beta-p} v(s)(\eta)\right\} .
$$

Suppose that $y \in B_{r}$ is arbitrary and let $\left\{v_{n}\right\}$ be a sequence in $S_{F, y}$. Then, by definition of $S_{F, y}$, we have $v_{n}(t) \in F(t, y(t))$ for almost all $t \in[0, T]$. Since $F(t, y(t))$ is compact for all $t \in J$, there is a convergent 
subsequence of $\left\{v_{n}(t)\right\}$ (we denote it by $\left\{v_{n}(t)\right\}$ again) that converges in measure to some $v(t) \in S_{F, y}$ for almost all $t \in J$. On the other hand, $\mathscr{L}$ is continuous, so $\mathscr{L}\left(v_{n}\right)(t) \rightarrow \mathscr{L}(v)(t)$ pointwise on $[0, T]$.

In order to show that the convergence is uniform, we have to show that $\left\{\mathscr{L}\left(v_{n}\right)\right\}$ is an equi-continuous sequence. Let $t_{1}, t_{2} \in[0, T]$ with $t_{1}<t_{2}$. It follows that

$$
\begin{aligned}
& \left|\mathscr{L}\left(v_{n}\right)\left(t_{2}\right)-\mathscr{L}\left(v_{n}\right)\left(t_{1}\right)\right| \\
\leq & \mid \frac{1}{\Gamma(\alpha+\beta)} \int_{0}^{t_{1}}\left[\left(t_{2}-s\right)^{\alpha+\beta-1}-\left(t_{1}-s\right)^{\alpha+\beta-1}\right] p(s) d s \\
& +\frac{1}{\Gamma(\alpha+\beta)} \int_{t_{1}}^{t_{2}}\left(t_{2}-s\right)^{\alpha+\beta-1} p(s) d s \mid \\
& +\frac{\left|t_{2}^{\beta}-t_{1}^{\beta}\right|}{|\Omega| \Gamma(\beta+1)}\left\{\sum_{i=1}^{n} \lambda_{i} \rho_{i} I_{\theta_{i}, \kappa_{i}}^{\gamma_{i}, \delta_{i}}\left(I^{\alpha+\beta} p(s)\right)\left(\xi_{i}\right)+I^{\alpha+\beta-\gamma} p(s)(\eta)\right\} .
\end{aligned}
$$

Obviously the right hand of the above inequality tends to zero as $t_{2} \rightarrow t_{1}$. Thus, the sequence $\left\{\mathscr{L}\left(v_{n}\right)\right\}$ is equi-continuous and hence, by the Arzelá-Ascoli theorem, we get that there is a uniformly convergent subsequence. So, there is a subsequence of $\left\{v_{n}\right\}$ (we denote it again by $\left\{v_{n}\right\}$ ) such that $\mathscr{L}\left(v_{n}\right) \rightarrow \mathscr{L}(v)$. Note that $\mathscr{L}(v) \in \mathscr{L}\left(S_{F, y}\right)$. Hence, $\mathscr{N}_{2}(y)=\mathscr{L}\left(S_{F, y}\right)$ is compact for all $y \in B_{r}$. So $\mathscr{N}_{2}(y)$ is compact.

Now, we show that $\mathscr{N}_{2}(y)$ is convex for all $y \in \mathscr{C}$. Let $z_{1}, z_{2} \in \mathscr{N}_{2}(y)$. We select $v_{1}, v_{2} \in S_{F, y}$ such that

$$
z_{i}(t)=I^{\alpha+\beta} v_{i}(s)(t)+\frac{t^{\beta}}{\Gamma(\beta+1)} \frac{1}{\Omega}\left\{\sum_{i=1}^{n} \lambda_{i}{ }^{\rho_{i}} I_{\theta_{i}, \kappa_{i}}^{\gamma_{i}, \delta_{i}}\left(I^{\alpha+\beta} v_{i}(s)\right)\left(\xi_{i}\right)-I^{\alpha+\beta-p} v_{i}(s)(\eta)\right\},
$$

$i=1,2$, for almost all $t \in[0, T]$. Let $0 \leq \lambda \leq 1$. Then, we have

$$
\begin{aligned}
{\left[\lambda z_{1}+(1-\lambda) z_{2}\right](t)=} & J^{\alpha}\left[\lambda v_{1}(s)+(1-\lambda) v_{2}(s)\right](t) \\
& +\frac{t^{\beta}}{\Gamma(\beta+1)} \frac{1}{\Omega}\left\{\sum_{i=1}^{n} \lambda_{i} \rho_{i} I_{\theta_{i}, \kappa_{i}}^{\gamma_{i}}\left(I^{\alpha+\beta}\left[\lambda v_{1}(s)+(1-\lambda) v_{2}(s)\right]\right)\left(\xi_{i}\right)\right. \\
& \left.-I^{\alpha+\beta-p}\left[\lambda v_{1}(s)+(1-\lambda) v_{2}(s)\right](\eta)\right\} .
\end{aligned}
$$

Since $F$ has convex values, so $S_{F, y}$ is convex and $\lambda v_{1}(s)+(1-\lambda) v_{2}(s) \in S_{F, y}$. Thus $\lambda z_{1}+(1-\lambda) z_{2} \in$ $\mathscr{N}_{2}(y)$. Consequently, $\mathscr{N}_{2}$ is convex-valued. Obviously, $\mathscr{N}_{1}$ is compact and convex-valued.

The rest of the proof consists of several steps and claims.

Step 1: We show that $\mathscr{N}_{1}$ is a contraction on $\mathscr{C}$. For $y, z \in \mathscr{C}$, we have

$$
\begin{aligned}
& \left|\mathscr{N}_{1} y(t)-\mathscr{N}_{1} z(t)\right| \\
= & \left|I^{\beta}\right| g(s, y(s))-g(s, z(s)) \mid(t) \\
& +\frac{t^{\beta}}{\Gamma(\beta+1)} \frac{1}{|\Omega|}\left\{\sum_{i=1}^{n} \lambda_{i} \rho_{i} I_{\theta_{i}, \kappa_{i}}^{\gamma_{i} \delta_{i}}\left(I^{\beta}|g(s, y(s))-g(s, z(s))|\right)\left(\xi_{i}\right)\right. \\
& \left.+I^{\beta-p}|g(s, y(s))-g(s, z(s))|(\eta)\right\} \\
\leq & k\left[\frac{T^{\beta}}{\Gamma(\beta+1)}+\frac{T^{\beta}}{|\Omega| \Gamma(\beta+1)}\left\{\sum_{i=1}^{n} \lambda_{i} \frac{\xi_{i}^{\kappa_{i}+\rho_{i}\left(\theta_{i}+\gamma_{i}\right)+\beta}}{\rho_{i}^{\delta_{i}}} \frac{\Gamma\left(\theta_{i}+\frac{\beta}{\rho_{i}}+1\right)}{\Gamma\left(\gamma_{i}+\theta_{i}+\frac{\beta}{\rho_{i}}+1\right)}\right.\right. \\
& \left.\left.+\frac{\eta^{\beta-p}}{\Gamma(\beta-p+1)}\right\}\right]\|y-z\|,
\end{aligned}
$$


which, on taking supremum over $t \in[0, T]$, yields

$$
\left\|\mathscr{N}_{1} y-\mathscr{N}_{1} z\right\| \leq k \Lambda_{1}\|x-y\| .
$$

This shows that $\mathscr{N}_{1}$ is a contraction as $k \Lambda_{1}<1$.

Step 2: $\mathscr{N}_{2}$ is compact and upper semicontinuous. This will be established in several claims.

CLAIM I: $\mathscr{N}_{2}$ maps bounded sets into bounded sets in $\mathscr{C}$.

Let $B_{r}=\{y \in \mathscr{C}:\|y\| \leq r\}$ be a bounded set in $\mathscr{C}$. Then, for each $h \in \mathscr{N}_{2}(y), y \in B_{r}$, there exists $v \in S_{F, y}$ such that

$$
h(t)=I^{\alpha+\beta} v(s)(t)+\frac{t^{\beta}}{\Gamma(\beta+1)} \frac{1}{\Omega}\left\{\sum_{i=1}^{n} \lambda_{i} \rho_{i} I_{\theta_{i}, \kappa_{i}}^{\gamma_{i}, \delta_{i}}\left(I^{\alpha+\beta} v(s)\right)\left(\xi_{i}\right)-I^{\alpha+\beta-p} v(s)(\eta)\right\} .
$$

Then, for $t \in[0, T]$, we have

$$
\begin{aligned}
& |h(t)| \\
\leq & I^{\alpha+\beta}|v(s)|(t)+\frac{t^{\beta}}{\Gamma(\beta+1)} \frac{1}{|\Omega|}\left\{\sum_{i=1}^{n} \lambda_{i} \rho_{i} I_{\theta_{i}, K_{i}}^{\gamma_{i} \delta_{i}}\left(I^{\alpha+\beta}|v(s)|\right)\left(\xi_{i}\right)+I^{\alpha+\beta-p}|v(s)|(\eta)\right\} \\
\leq & \|p\|\left[\frac{T^{\alpha+\beta}}{\Gamma(\alpha+\beta+1)}+\frac{T^{\beta}}{|\Omega| \Gamma(\beta+1)}\left\{\sum_{i=1}^{n} \lambda_{i} \frac{\xi_{i}^{\kappa_{i}+\rho_{i}\left(\theta_{i}+\gamma_{i}\right)+\alpha+\beta}}{\rho_{i}^{\delta_{i}}} \frac{\Gamma\left(\theta_{i}+\frac{\alpha+\beta}{\rho_{i}}+1\right)}{\Gamma\left(\gamma_{i}+\theta_{i}+\frac{\alpha+\beta}{\rho_{i}}+1\right)}\right.\right. \\
& \left.\left.+\frac{\eta^{\alpha+\beta-p}}{\Gamma(\alpha+\beta-p+1)}\right\}\right] .
\end{aligned}
$$

Thus,

$$
\|h\| \leq\|p\| \Lambda_{2} .
$$

CLAIM II: $\mathscr{N}_{2}$ maps bounded sets into equi-continuous sets.

Let $t_{1}, t_{2} \in[0, T]$ with $t_{1}<t_{2}$ and $y \in B_{r}$. Then, for each $h \in \mathscr{N}_{2}(y)$, we obtain

$$
\begin{aligned}
& \left|h\left(t_{2}\right)-h\left(t_{1}\right)\right| \\
\leq & \mid \frac{1}{\Gamma(\alpha+\beta)} \int_{0}^{t_{1}}\left[\left(t_{2}-s\right)^{\alpha+\beta-1}-\left(t_{1}-s\right)^{\alpha+\beta-1}\right] v(s) d s \\
& +\frac{1}{\Gamma(\alpha+\beta)} \int_{t_{1}}^{t_{2}}\left(t_{2}-s\right)^{\alpha+\beta-1} v(s) d s \mid \\
& +\frac{\left|t_{2}^{\beta}-t_{1}^{\beta}\right|}{|\Omega| \Gamma(\beta+1)}\left\{\sum_{i=1}^{n} \lambda_{i} \rho_{i} I_{\theta_{i}, \kappa_{i}, \delta_{i}}\left(I^{\alpha+\beta}|v(s)|\right)\left(\xi_{i}\right)+I^{\alpha+\beta-\gamma}|v(s)|(\eta)\right\} \\
\leq & \frac{\|p\|}{\Gamma(\alpha+\beta+1)}\left[t_{2}^{\alpha+\beta}-t_{1}^{\alpha+\beta}+2\left(t_{2}-t_{1}\right)^{\alpha+\beta}\right] \\
& +\frac{\left|t_{2}^{\beta}-t_{1}^{\beta}\right|\|p\| \mid}{|\Omega| \Gamma(\beta+1)}\left\{\sum_{i=1}^{n} \lambda_{i} \frac{\xi_{i}^{\kappa_{i}+\rho_{i}\left(\theta_{i}+\gamma_{i}\right)+\alpha+\beta}}{\rho_{i}^{\delta_{i}}} \frac{\Gamma\left(\theta_{i}+\frac{\alpha+\beta}{\rho_{i}}+1\right)}{\Gamma\left(\gamma_{i}+\theta_{i}+\frac{\alpha+\beta}{\rho_{i}}+1\right)}+\frac{\eta^{\alpha+\beta-p}}{\Gamma(\alpha+\beta-p+1)}\right\} .
\end{aligned}
$$

Obviously the right hand side of the above inequality tends to zero independently of $y \in B_{r}$ as $t_{2}-t_{1} \rightarrow 0$. Therefore it follows by the Ascoli-Arzelá theorem that $\mathscr{N}_{2}: \mathscr{C} \rightarrow \mathscr{P}(\mathscr{C})$ is completely continuous.

By Claims I and II, $\mathscr{N}_{2}$ is completely continuous. By Lemma $5.2, \mathscr{N}_{2}$ will be upper semicontinuous (since it is completely continuous) if we prove that it has a closed graph. 
CLAIM III: $\mathscr{N}_{2}$ has a closed graph.

Let $y_{n} \rightarrow x_{*}, h_{n} \in \mathscr{N}_{2}\left(y_{n}\right)$ and $h_{n} \rightarrow h_{*}$. Then we need to show that $h_{*} \in \mathscr{N}_{2}\left(y_{*}\right)$. Associated with $h_{n} \in \mathscr{N}_{2}\left(y_{n}\right)$, there exists $v_{n} \in S_{F, y_{n}}$ such that for each $t \in[0, T]$,

$$
h_{n}(t)=I^{\alpha+\beta} v_{n}(s)(t)+\frac{t^{\beta}}{\Gamma(\beta+1)} \frac{1}{\Omega}\left\{\sum_{i=1}^{n} \lambda_{i} \rho_{i} I_{\theta_{i}, \kappa_{i}}^{\gamma_{i}, \delta_{i}}\left(I^{\alpha+\beta} v_{n}(s)\right)\left(\xi_{i}\right)-I^{\alpha+\beta-p} v_{n}(s)(\eta)\right\} .
$$

Thus it suffices to show that there exists $v_{*} \in S_{F, y_{*}}$ such that, for each $t \in[0, T]$,

$$
h_{*}(t)=I^{\alpha+\beta} v_{*}(s)(t)+\frac{t^{\beta}}{\Gamma(\beta+1)} \frac{1}{\Omega}\left\{\sum_{i=1}^{n} \lambda_{i} \rho_{i} I_{\theta_{i}, \kappa_{i}}^{\gamma_{i}} \delta_{i}\left(I^{\alpha+\beta} v_{*}(s)\right)\left(\xi_{i}\right)-I^{\alpha+\beta-p} v_{*}(s)(\eta)\right\} .
$$

Let us consider the linear operator $\Theta: L^{1}([0, T], \mathbb{R}) \rightarrow \mathscr{C}$ given by

$$
v \mapsto \Theta(v)(t)=I^{\alpha+\beta} v(s)(t)+\frac{t^{\beta}}{\Gamma(\beta+1)} \frac{1}{\Omega}\left\{\sum_{i=1}^{n} \lambda_{i} \rho_{i} I_{\theta_{i}, \kappa_{i}}^{\gamma_{i}, \delta_{i}}\left(I^{\alpha+\beta} v(s)\right)\left(\xi_{i}\right)-I^{\alpha+\beta-p} v(s)(\eta)\right\} .
$$

Observe that $\left\|h_{n}(t)-h_{*}(t)\right\| \rightarrow 0$, as $n \rightarrow \infty$. Thus, it follows by Lemma 5.3 that $\Theta \circ S_{F}$ is a closed graph operator. Further, we have $h_{n}(t) \in \Theta\left(S_{F, y_{n}}\right)$. Since $y_{n} \rightarrow y_{*}$, we have that

$$
h_{*}(t)=I^{\alpha+\beta} v_{*}(s)(t)+\frac{t^{\beta}}{\Gamma(\beta+1)} \frac{1}{\Omega}\left\{\sum_{i=1}^{n} \lambda_{i} \rho_{i} I_{\theta_{i}, \kappa_{i}}^{\gamma_{i}, \delta_{i}}\left(I^{\alpha+\beta} v_{*}(s)\right)\left(\xi_{i}\right)-I^{\alpha+\beta-p} v_{*}(s)(\eta)\right\},
$$

for some $v_{*} \in S_{F, y_{*}}$. Hence $\mathscr{N}_{2}$ has a closed graph (and therefore has closed values). In consequence, the operator $\mathscr{N}_{2}$ is compact and upper semicontinuous.

Step 3: Here, we show that $\mathscr{N}_{1}(y)+\mathscr{N}_{2}(y) \subset B_{r}$ for all $y \in B_{r}$. Suppose $y \in B_{r}$, with

$$
r>\frac{g_{0} \Lambda_{1}+\|p\| \Lambda_{2}}{1-k \Lambda_{1}}
$$

and $h \in \mathscr{N}_{2}$ are arbitrary elements. Choose $v \in S_{F, y}$ such that

$$
\begin{aligned}
h(t)= & I^{\beta} g(s, y(s))(t)+I^{\alpha+\beta} v(s)(t) \\
& +\frac{t^{\beta}}{\Gamma(\beta+1)} \frac{1}{\Omega}\left\{\sum_{i=1}^{n} \lambda_{i}{ }^{\rho_{i}} I_{\theta_{i}, \kappa_{i}}^{\gamma_{i}, \delta_{i}}\left(I^{\beta} g(s, y(s))+I^{\alpha+\beta} v(s)\right)\left(\xi_{i}\right)\right. \\
& \left.-I^{\beta-p} g(s, y(s))(\eta)-I^{\alpha+\beta-p} v(s)(\eta)\right\} .
\end{aligned}
$$

Following the method for proof for Claim I, and using the fact that $|g(t, y(t))| \leq|g(t, y(t))-g(t, 0)|+$ $|g(t, 0)| \leq k r+g_{0}, g_{0}=\sup _{t \in[0, T]}|g(t, 0)|$, we can obtain

$$
\begin{aligned}
|h(t)| \leq & \left(k r+g_{0}\right)\left[\frac{T^{\beta}}{\Gamma(\beta+1)}+\frac{T^{\beta}}{|\Omega| \Gamma(\beta+1)}\left\{\sum_{i=1}^{n} \lambda_{i} \frac{\xi_{i}^{\kappa_{i}+\rho_{i}\left(\theta_{i}+\gamma_{i}\right)+\beta}}{\rho_{i}^{\delta_{i}}} \frac{\Gamma\left(\theta_{i}+\frac{\beta}{\rho_{i}}+1\right)}{\Gamma\left(\gamma_{i}+\theta_{i}+\frac{\beta}{\rho_{i}}+1\right)}\right.\right. \\
& \left.+\frac{\eta^{\beta-p}}{\Gamma(\beta-p+1)}\right\}+\|p\|\left[\frac{T^{\alpha+\beta}}{\Gamma(\alpha+\beta+1)}\right. \\
& +\frac{T^{\beta}}{|\Omega| \Gamma(\beta+1)}\left\{\sum_{i=1}^{n} \lambda_{i} \frac{\xi_{i}^{\kappa_{i}+\rho_{i}\left(\theta_{i}+\gamma_{i}\right)+\alpha+\beta}}{\rho_{i}^{\delta_{i}}} \frac{\Gamma\left(\theta_{i}+\frac{\alpha+\beta}{\rho_{i}}+1\right)}{\Gamma\left(\gamma_{i}+\theta_{i}+\frac{\alpha+\beta}{\rho_{i}}+1\right)}\right. \\
& \left.\left.+\frac{\eta^{\alpha+\beta-p}}{\Gamma(\alpha+\beta-p+1)}\right\}\right] .
\end{aligned}
$$


Thus

$$
\|h\| \leq\left(k r+g_{0}\right) \Lambda_{1}+\|p\| \Lambda_{2}<r .
$$

Hence $\|h\| \leq r$, which means that $\mathscr{N}_{1}(y)+\mathscr{N}_{2}(y) \subset B_{r}$ for all $y \in B_{r}$. Thus, the operators $\mathscr{N}_{1}$ and $\mathscr{N}_{2}$ satisfy all the conditions of Lemma 5.4 and hence its conclusion implies that $y \in \mathscr{N}_{1}(y)+\mathscr{N}_{2}(y)$ in $B_{r}$. Therefore the boundary value problem (1.2) has a solution in $B_{r}$ and the proof is completed.

To prove our next result, we make use of the following form of the nonlinear alternative for contractive maps [31, Corollary 3.8].

Theorem 5.7. Let $X$ be a Banach space, and $D$ a bounded neighborhood of $0 \in X$. Let $Z_{1}: X \rightarrow \mathscr{P}_{c p, c}(X)$ and $Z_{2}: \bar{D} \rightarrow \mathscr{P}_{c p, c}(X)$ two multi-valued operators satisfying

(a) $Z_{1}$ is contraction, and

(b) $Z_{2}$ is u.s.c and compact.

Then, if $G=Z_{1}+Z_{2}$, either

(i) G has a fixed point in $\bar{D}$ or

(ii) there is a point $u \in \partial D$ and $\lambda \in(0,1)$ with $u \in \lambda G(u)$.

Theorem 5.8. Assume that $\left(A_{1}\right)$ with $k<\Lambda_{1}^{-1}$ and $\left(H_{1}\right)$ are satisfied. In addition we suppose that:

$\left(C_{1}\right)$ there exists a continuous nondecreasing function $\psi:[0, \infty) \rightarrow(0, \infty)$ and a function $p \in C\left([0, T], \mathbb{R}^{+}\right)$ such that

$$
\|F(t, x)\|_{\mathscr{P}}:=\sup \{|y|: y \in F(t, x)\} \leq p(t) \psi(\|x\|) \text { for each }(t, x) \in[0, T] \times \mathbb{R}
$$

$\left(C_{2}\right)$ there exists a number $M>0$ such that

$$
\frac{\left(1-k \Lambda_{1}\right) M}{\Lambda_{2}\|p\| \psi(M)+\Lambda_{1} g_{0}}>1
$$

where $\Lambda_{1}, \Lambda_{2}$ are defined by (3.2) and (3.3) respectively.

Then boundary value problem (1.2) has at least one solution on $[0, T]$.

Proof. As in Theorem 5.6, one can show that operators $\mathscr{N}_{1}$ and $\mathscr{N}_{2}$ defined by (5.2) and (5.3) respectively are indeed the multivalued operators $\mathscr{N}_{1}, \mathscr{N}_{2}: B_{r} \rightarrow \mathscr{P}_{c p, c}(\mathscr{C})$, where $B_{r}=\{y \in \mathscr{C}:\|y\| \leq r\}$ is a bounded set in $\mathscr{C}, \mathscr{N}_{1}$ is a contraction on $\mathscr{C}$ and $\mathscr{N}_{2}$ is u.s.c. and compact. Thus $\mathscr{N}_{1}$ and $\mathscr{N}_{2}$ satisfy all the conditions of Theorem 5.7 and hence its conclusion implies either condition (i) or condition (ii) holds. We show that the conclusion (ii) is not possible. If $y \in \lambda\left(\mathscr{N}_{1}(y)+\mathscr{N}_{2}(y)\right)$ for $\lambda \in(0,1)$, then there exists $v \in S_{F, y}$ such that

$$
\begin{aligned}
y(t)= & I^{\beta} g(s, y(s))(t)+I^{\alpha+\beta} v(s)(t) \\
& +\frac{t^{\beta}}{\Gamma(\beta+1)} \frac{1}{\Omega}\left\{\sum_{i=1}^{n} \lambda_{i}{ }^{\rho_{i}} I_{\theta_{i}, \kappa_{i}}^{\gamma_{i}, \delta_{i}}\left(I^{\beta} g(s, y(s))+I^{\alpha+\beta} v(s)\right)\left(\xi_{i}\right)\right. \\
& \left.-I^{\beta-p} g(s, y(s))(\eta)-I^{\alpha+\beta-p} v(s)(\eta)\right\}, \quad t \in[0, T],
\end{aligned}
$$


and

$$
\begin{aligned}
& |y(t)| \\
& \leq I^{\beta}|g(s, y(s))|(t)+I^{\alpha+\beta}|v(s)|(t)+\frac{T^{\beta}}{|\Omega| \Gamma(\beta+1)}\left\{\sum_{i=1}^{n} \lambda_{i} \rho_{i} I_{\theta_{i}, \kappa_{i}}^{\gamma_{i}, \delta_{i}}\left(I^{\beta}|g(s, y(s))|\right)\left(\xi_{i}\right)\right. \\
& \left.+\sum_{i=1}^{n} \lambda_{i} \rho_{i} I_{\theta_{i}, \kappa_{i}}^{\gamma_{i}, \delta_{i}}\left(I^{\alpha+\beta}|v(s)|\right)\left(\xi_{i}\right)+I^{\beta-p}|g(s, y(s))|(\eta)+I^{\alpha+\beta-p}|v(s)|(\eta)\right\} \\
& \leq\left(k\|y\|+g_{0}\right) \frac{T^{\beta}}{\Gamma(\beta+1)}+\|p\| \psi(\|y\|) \frac{T^{\alpha+\beta}}{\Gamma(\alpha+\beta+1)} \\
& +\frac{T^{\beta}}{|\Omega| \Gamma(\beta+1)}\left\{\sum_{i=1}^{n} \lambda_{i}\left(k\|y\|+g_{0}\right) \frac{\xi_{i}^{\kappa_{i}+\rho_{i}\left(\theta_{i}+\gamma_{i}\right)+\beta}}{\rho_{i}^{\delta_{i}}} \frac{\Gamma\left(\theta_{i}+\frac{\beta}{\rho_{i}}+1\right)}{\Gamma\left(\gamma_{i}+\theta_{i}+\frac{\beta}{\rho_{i}}+1\right)}\right. \\
& +\sum_{i=1}^{n} \lambda_{i}\|p\| \psi(\|y\|) \frac{\xi_{i}^{\kappa_{i}+\rho_{i}\left(\theta_{i}+\gamma_{i}\right)+\alpha+\beta}}{\rho_{i}^{\delta_{i}}} \frac{\Gamma\left(\theta_{i}+\frac{\alpha+\beta}{\rho_{i}}+1\right)}{\Gamma\left(\gamma_{i}+\theta_{i}+\frac{\alpha+\beta}{\rho_{i}}+1\right)} \\
& \left.+\left(k\|y\|+g_{0}\right) \frac{\eta^{\beta-p}}{\Gamma(\beta-p+1)}+\|p\| \psi(\|y\|) \frac{\eta^{\alpha+\beta-p}}{\Gamma(\alpha+\beta-p+1)}\right\} \\
& \leq \Lambda_{1}\left(k\|y\|+g_{0}\right)+\Lambda_{2}\|p\| \psi(\|y\|) \text {. }
\end{aligned}
$$

Thus

$$
\left(1-k \Lambda_{1}\right)\|y\| \leq \Lambda_{2}\|p\| \psi(\|y\|)+\Lambda_{1} g_{0} .
$$

If condition (ii) of Theorem 5.7 holds, then there exists $\lambda \in(0,1)$ and $y \in \partial B_{M}$ with $y=\lambda \mathscr{N}(y)$. Then, $y$ is a solution of (1.2) with $\|y\|=M$. Now, by the inequality (5.6), we get

$$
\frac{\left(1-k \Lambda_{1}\right) M}{\Lambda_{2}\|p\| \psi(M)+\Lambda_{1} g_{0}} \leq 1
$$

which contradicts (5.5). Hence, $\mathscr{N}$ has a fixed point in $[0, T]$ by Theorem 5.7, and consequently problem (1.2) has a solution. This completes the proof.

\section{Discussion}

We have derived existence and uniqueness results for neutral Caputo type fractional differential equations supplemented with generalized nonlocal fractional integro-differential boundary conditions. Our results are quite general and give rise to several special cases for different values of the parameters involved in the given problem. We enlist some special cases below, see [32].

(i) If $\kappa=0, \theta=0$ and $\rho \rightarrow 1$, then our results correspond to the ones for nonlocal fractional Riemann-Liouville type integro-differential boundary conditions.

(ii) If $\delta=\gamma, \kappa=0, \theta=0$, and $\rho \rightarrow 0+$, then we obtain the results for nonlocal fractional Hadamard type integro-differential boundary conditions. Notice that the generalized integral in the boundary conditions reduces to the Hadamard fractional integral of order $\gamma$ :

$$
H^{\gamma} f(t)=\frac{1}{\Gamma(\gamma)} \int_{0}^{t}\left(\log \frac{t}{s}\right)^{\gamma-1} \frac{f(s)}{s} d s,
$$

in the limit $\rho \rightarrow 0+$ with the aid of L'Hospital's rule. 
(iii) If $\delta=0$ and $\kappa=-\rho(\gamma+\theta)$, then the generalized integral in the boundary conditions becomes Erdélyi-Kober fractional integral

$$
I_{\theta,-\rho(\gamma+\theta)}^{\gamma} f(t)=\frac{\rho t^{-\rho(\gamma+\theta)}}{\Gamma(\gamma)} \int_{0}^{t} \frac{s^{\rho(\theta+1)-1}}{\left(t^{\rho}-s^{\rho}\right)^{1-\gamma}} f(s) d s .
$$

(iv) If $\delta=\gamma, \kappa=0$ and $\theta=0$, then the integral operator in the boundary conditions takes the form of Katugampola fractional integral

$$
\rho^{\gamma} I^{\gamma} f(t)=\frac{\rho^{1-\gamma}}{\Gamma(\gamma)} \int_{0}^{t} \frac{s^{\rho-1}}{\left(t^{\rho}-s^{\rho}\right)^{1-\gamma}} f(s) d s .
$$

\section{REFERENCES}

[1] A.A. Kilbas, H.M. Srivastava, J.J. Trujillo, Theory and Applications of Fractional Differential Equations, North-Holland Mathematics Studies, 204. Elsevier Science B.V., Amsterdam, 2006.

[2] V. Lakshmikantham, S. Leela, J.V. Devi, Theory of Fractional Dynamic Systems, Cambridge Academic Publishers, Cambridge, 2009.

[3] I. Podlubny, Fractional Differential Equations, Academic Press, San Diego, 1999.

[4] K. Diethelm, The Analysis of Fractional Differential Equations, Lecture Notes in Mathematics, Springer-verlag Berlin Heidelberg, 2010.

[5] B. Ahmad, A. Alsaedi, S.K. Ntouyas, J. Tariboon, Hadamard-type Fractional Differential Equations, Inclusions and Inequalities, Springer, Berlin, 2017.

[6] R.P. Agarwal, Y. Zhou, J.R. Wang, X. Luo, Fractional functional differential equations with causal operators in Banach spaces, Math. Comput. Modelling 54 (2011), 1440-1452.

[7] S. Abbas, M. Benchohra, J.E. Lazreg, Y. Zhou, A survey on Hadamard and Hilfer fractional differential equations: analysis and stability, Chaos, Solitons Fractals 102 (2017), 47-71.

[8] B. Ahmad, S.K. Ntouyas, A. Alsaedi, New existence results for nonlinear fractional differential equations with three-point integral boundary conditions, Adv. Difference Equ. 2011 (2011), Article ID 107384.

[9] B. Ahmad, S.K. Ntouyas, Existence results for a coupled system of Caputo type sequential fractional differential equations with nonlocal integral boundary conditions, Appl. Math. Comput. 266 (2015), 615-622.

[10] B. Ahmad, S.K. Ntouyas, J. Tariboon, A study of mixed Hadamard and Riemann-Liouville fractional integro-differential inclusions via endpoint theory, Appl. Math. Lett. 52 (2016), 9-14.

[11] B. Ahmad, S.K. Ntouyas, A. Alsaedi, Caputo-type fractional boundary value problems for differential equations and inclusions with multiple fractional derivatives, J. Nonlinear Funct. Anal. 2017 (2017), Article ID 52.

[12] B. Ahmad, S.K. Ntouyas, Existence results for fractional differential inclusions with Erdelyi-Kober fractional integral conditions, An. Ştiinţ. Univ. Ovidius Constanţa Ser. Mat. 25 (2017), 5-24.

[13] M. Benchohra, J. Henderson, S.K. Ntouyas, A. Ouahab, Existence results for fractional order functional differential equations with infinite delay, J. Math. Anal. Appl. 338 (2008), 1340-1350.

[14] H. Ergören, B. Ahmad, Neutral functional fractional differential inclusions with impulses at variable times, Dyn. Contin. Discrete Impuls. Syst. Ser. B Appl. Algorithms 24 (2017), 235-246.

[15] D. Qarout, B. Ahmad, A. Alsaedi, Existence theorems for semi-linear Caputo fractional differential equations with nonlocal discrete and integral boundary conditions, Fract. Calc. Appl. Anal 19 (2016), 463-479.

[16] X. Su, M. Jia, X. Fu, On positive solutions of eigenvalue problems for a class of $p$-Laplacian fractional differential equations, J. Appl. Anal. Comput. 8 (2018), 152-171.

[17] C. Yu, G. Gao, Some results on a class of fractional functional differential equations, Commun. Appl. Nonlinear Anal. 11 (2004), 67-75.

[18] C. Yu, G. Gao, Existence of fractional differential equations, J. Math. Anal. Appl. 310 (2005), 26-29.

[19] Y. Zhou, F. Jiao, J. Pecaric, Abstract Cauchy problem for fractional functional differential equations, Topol. Methods Nonlinear Anal. 42 (2013), 119-136.

[20] U. N. Katugampola, New fractional integral unifying six existing fractional integrals, (2016), arxiv:1612.08596. 
[21] B. Ahmad, A. Alsaedi, B.S. Alghamdi, Analytic approximation of solutions of the forced Duffing equation with integral boundary conditions, Nonlinear Anal. Real World Appl. 9 (2008), 1727-1740.

[22] R. Ciegis, A. Bugajev, Numerical approximation of one model of the bacterial self-organization, Nonlinear Anal. Model. Control 17 (2012), 253-270.

[23] A. Bitsadze, A. Samarskii, On some simple generalizations of linear elliptic boundary problems, Russian Acad. Sci. Dokl. Math. 10 (1969), 398-400.

[24] A. Granas, J. Dugundji, Fixed Point Theory, Springer-Verlag, New York, 2003.

[25] M.A. Krasnoselskii, Two remarks on the method of successive approximations, Uspekhi Matematicheskikh Nauk 10 (1955), 123-127.

[26] K. Deimling, Multivalued Differential Equations, Walter De Gruyter, Berlin-New York, 1992.

[27] S. Hu, N. Papageorgiou, Handbook of Multivalued Analysis, vol. 1. Kluwer, Dordrecht, 1997.

[28] G.V. Smirnov, Introduction to the Theory of Differential Inclusions, American Mathematical Society, Providence, RI, 2002.

[29] A. Lasota, Z. Opial, An application of the Kakutani-Ky-Fan theorem in the theory of ordinary differential equations or noncompact acyclic-valued map, Bull. Acad. Pol. Sci. Ser. Sci. Math. Astronom. Phys. 13 (1965), 781-786.

[30] A. Petrusel, Fixed points and selections for multivalued operators, Seminar on Fixed Point Theory Cluj-Napoca 2 (2001), 3-22.

[31] W.V. Petryshyn, P. M. Fitzpatric, A degree theory, fixed point theorems, and mapping theorems for multivalued noncompact maps, Trans. Amer. Math. Soc. 194 (1974), 1-25.

[32] J. Vanterler da C. Sousa, D.S. Oliveira, E. Capelas de Oliveira, Grüss-type inequality by mean of a fractional integral, (2017), arXiv:1705.00965. 